\title{
Integrins and focal adhesion kinase in the malignant behavior of gliomas
}

\author{
Efstathia Giannopoulou ${ }^{1}$, Andreas Tzakos ${ }^{2}$, Andreas A. Argyriou ${ }^{3}$ \\ ${ }^{1}$ Clinical Oncology Laboratory, Division of Oncology, Department of Medicine, University of Patras, 26504 Patras, Greece. \\ ${ }^{2}$ Section of Organic Chemistry and Biochemistry, Department of Chemistry, University of Ioannina, 45110 Ioannina, Greece. \\ ${ }^{3}$ Department of Neurology, "Saint Andrew's" General Hospital, 26335 Patras, Greece.
}

\section{A B S T R A C T}

Glioblastoma multiforme (GBM) is the most common type of glioma and is associated with a very poor prognosis. The standard treatment includes radiotherapy concurrent with temozolomide, however recently the Food and Drug Administration approved bevacizumab for use in patients with progressive glioblastoma following prior therapy. The limited number of treatment options points to the need for novel effective therapeutic approaches. A promising approach is the use of tyrosine kinase inhibitors (TKIs) in GBM treatment. However, the results from the majority of clinical trials using TKIs are not very encouraging. One growing area is the development of tumor-homing peptides that resemble the integrin recognition sequence RGD. In this article, the role of integrins and focal adhesion kinase in malignant glioma is reviewed, and an experimental study is proposed that will apply a strategy for peptide-mediated delivery of compounds deep into tumor parenchyma using tumor-homing peptides.

Key words: Focal adhesion kinase, glioblastoma multiforme, integrins

\section{INTRODUCTION}

Malignant glioma (MG), including glioblastoma multiforme (GBM) and anaplastic astrocytoma, ranks among the most common primary brain tumors. Apart from maximally safe surgical resection, the first-line treatment consists of radiotherapy and concomitant systemic application of chemotherapy-usually with temozolomide-following the Stupp regimen, which represents the standard conventional treatment for GBM. Nonetheless, the median survival time of patients with MGs, and consequently their outcome, remains very poor. ${ }^{[1-3]}$

Several mechanisms of GBM resistance to standard chemotherapy have been proposed. The use of chemotherapy has been reported as being limited, due to the fact that the blood-brain barrier restricts the accumulation of conventional cytotoxic agents to therapeutic concentrations in the tumor and the

\begin{tabular}{|l|l|}
\hline \multicolumn{2}{|c|}{ Access this article online } \\
\hline Quick Response Code: & \\
\hline & Website: \\
\hline
\end{tabular}

peritumoral area. ${ }^{[2-4]}$ Other restricting factors include potential interactions between antiepileptic drugs and chemotherapeutic agents, use of glucocorticosteroids, and the implication of specific genetic transformation and characteristics of GBMs. In particular, the multidrug resistance system is considered to be mainly responsible for the development of treatment resistance. ${ }^{[4]}$

\section{PATHOGENETIC ASPECTS OF GLIOBLASTOMA MULTIFORMES}

The pathological hallmarks of GBMs include rapid progression, neovascularization, necrosis, and intense apoptotic resistance. Common genetic alterations associated with malignant phenotypic characteristics are commonly found in tumors. However, the molecular mechanisms leading to these phenotypic features are as yet vaguely defined, mainly due to genetic heterogeneity, even within the same tumor. ${ }^{[5]}$ However, there are some known mutations, deletions, or alterations in gene expression that have been linked to the genesis of GBMs..$^{[3,6]}$ Several signaling pathways leading to malignant behavior in MGs, induction of cell migration, and tumor invasiveness have also been implicated. These pathways are regulated by amplification and/or overexpression of several growth factor receptors linked with tyrosine kinases, such as the epidermal growth factor receptor (EGFR), insulin-like

Corresponding Author: Dr. Efstathia Giannopoulou, Clinical Oncology Laboratory, Division of Oncology, Department of Medicine, University of Patras, 26504 Patras, Greece. E-mail: giannop@upatras.gr 
growth factor receptor, platelet-derived growth factor receptor (PDGFR), vascular endothelial growth factor receptor (VEGFR), and hepatocyte growth factor/scatter factor receptor. Several hormones and cytokines are also involved in the regulation of molecular pathways related to GBM development. ${ }^{[5]}$ Recent data implicate the inflammatory interleukins (IL)-1 $\beta,-6$ and -8 in GBM pathophysiology. It has been found that these cytokines are upregulated in GBM cell lines as well as in patients' samples while some of them have high prognostic potential. ${ }^{[7]}$

Angiogenesis is considered to be the key regulating factor of vascular development in tumors and especially for GBMs. The development and growth of MGs seem to be dependent on angiogenesis since microvascular proliferation can only be observed in high-grade gliomas. ${ }^{[2,8-10]}$ Apart from growth factors and their receptors, other molecules that significantly contribute to angiogenesis in gliomas and growth of GBMs are the integrins and focal adhesion kinase (FAK).

\section{INTEGRINS}

Integrins are cell surface receptor glycoproteins, mediating various intracellular signals through interaction with the extracellular matrix (ECM). Integrins also significantly contribute to the attachment of cells to the ECM through the formation of cell adhesion complexes consisting of integrins and cytoplasmic proteins. There are many types of integrins, which are obligate heterodimers containing two distinct chains called $\alpha$ and $\beta$ subunits. The combination of $\alpha$ and $\beta$ subunits determines the ligand specificity. ${ }^{[11-13]}$

Integrins are crucial molecules in glioma because of their contribution to enhanced invasion capacity in glioma cells. This phenotype can be defined by three attributes. The cells at the invasive edge of the tumor are able to: (i) detach and migrate forward; (ii) adhere via local and self-produced ECM; and (iii) degrade the local/surrounding ECM in order to clear a path for further invasion. Since integrins are integral to the process of cell adhesion and migration, these receptors have been assessed as potential contributors to glioma invasion, as have been the cooperating ECM components. ${ }^{[11]}$ Multiple integrins have been reported to be expressed on GBM in tissue biopsies, including $\alpha_{v} \beta_{3}, \alpha_{v} \beta_{5}, \alpha_{5} \beta_{1}, \alpha_{2} \beta_{1}, \alpha_{3} \beta_{1}, \alpha_{6} \beta_{1}$, and $\alpha_{v} \beta_{1}$.

Functional studies with blocking antibodies directed toward the $\beta_{1}$ integrin subunit have shown an inhibition of adhesion, motility, and invasion of cultured glioma cells plated on multiple ECM substrates (laminin, collagen type IV, fibronectin, and vitronectin), suggesting a role for one or more $\beta_{1}$ integrins in neoplastic glial cell migration into the brain. ${ }^{[12]}$ Another study utilizing neutralizing antibodies directed toward the $\alpha_{v}$ or the $\beta_{1}$ integrin subunits reported complete inhibition of GBM cell migration by most substrates, suggesting the $\alpha_{\mathrm{v}}$ and $\beta_{1}$ integrins play a crucial role in GBM tumor cell infiltration into the normal brain. ${ }^{[12]}$

Integrins modulate several functions of GBM cells-including survival, adhesion, and migrationthrough interaction between growth factors and their receptors with subsequent formation of complexes. There is robust evidence suggesting the formation of analogous complexes in different types of cells, such as complexes of various types of integrins with VEGFR-2, PDGFR- $\beta$, and EGFR. ${ }^{[14,15]}$

\section{FOCAL ADHESION KINASE}

Focal adhesion kinase has been recently established as a key component of the signal transduction pathways triggered by integrins. FAK not only acts directly on the plasticity of cytoskeletal structures at focal adhesions, but also mediates effects on gene expression that indirectly alter the ability of cells to migrate and invade. ${ }^{[13]}$ The interaction of urokinase-type plasminogen activator receptor (uPAR) with integrins during cell adhesion and migration has also been proposed. uPAR binds the urokinase-type plasminogen activator (UPA) and facilitates a proteolytic cascade focused on the cell surface. uPAR has recently been recognized as a multifunctional protein that, through its interactions with integrins, initiates signaling events that alter cell adhesion, migration, and proliferation of various cancer cells, including GBM cells. ${ }^{[11,12,16]}$

All these molecular and genetic alterations contribute to the well-established biological features of GBMs and may provide a target to enhance therapeutic responsiveness of these lethal brain malignancies. Recent advances in thorough understanding of the complex molecular pathogenesis of GBMs have led to the rational development of new treatment options targeting intracellular signaling. ${ }^{[1,2]}$ Despite these advances, most single-agent therapies targeted to growth and survival pathways have failed to demonstrate a significant survival benefit, mainly because of the complexity of the implicated signaling pathways and their interactions. Thus, targeting multiple signaling pathways by multi-target kinase inhibitors or combinations of single-target kinase inhibitors may increase treatment efficacy. Multi-targeted agents are needed to simultaneously target multiple signaling pathways that occur either at the same time or sequentially, as a compensatory mechanism to tumor growth and resistance to treatment. ${ }^{[1]}$ Currently, several 
multi-target kinase inhibitors and combinations of single-targeted kinase inhibitors that simultaneously affect multiple pathways such as signaling, repair, and angiogenesis have been tested in clinical trials for their ability to effectively prolong the median survival time of patients and to establish future directions in GBM therapy. Overall, the identification of new targeted strategies for GBMs remains a very challenging area in the field, since it has the potential to positively affect patient outcome, survival rate, and quality of life.

Preclinical, as well as clinical studies with various integrin antagonists, have demonstrated their effectiveness in blocking tumor progression. Phase II clinical trials with cilengitide (Merck KGaA, Darmstadt, Germany), an $\alpha_{v} \beta_{3}$ and $\alpha_{v} \beta_{5}$ integrin antagonists, have shown clinical activity and few side effects in patients with glioblastoma. ${ }^{[17]}$ Cilengitide is a synthetic Arg-Gly-Asp (RGD) pentapeptide recognizing the RGD ligand binding motif on the integrin receptors $\alpha_{v} \beta_{3}$ and $\alpha_{v} \beta_{5}^{[18]}$ and competitively blocks integrin ligand binding. It has been shown to diminish angiogenesis in vitro. ${ }^{[19]}$ In an important early preclinical study, cilengitide markedly suppressed tumor growth in amedulloblastoma and orthotopic glioblastoma models (i.e., when tumors were grown in the brain), whereas no growth inhibition was demonstrated in a heterotopic model (i.e., when tumors were grown in the flank of nude mice) or when an inactive peptide was used. ${ }^{[20]}$ This suggests that the brain environment is particularly susceptible to the integrin inhibition and has led to subsequent clinical investigation. ${ }^{[21]}$

In addition, a new variant of RGD (internalizing RGD, iRGD) that combines the RGD motif with a tissue penetration element called C-end rule (CendR) has been recently presented. ${ }^{[22]}$ Like the earlier RGD peptides, iRGD homes to tumors, but exposure of the CendR motif activates a transport system through tumor blood vessel walls into the tumor core. Interestingly, it was shown that coupling of iRGD to anti-cancer drugs allowed them to penetrate deeply into tumors, effectively increasing the activity of the drugs. ${ }^{[22]}$ More specifically, intravenously injected compounds coupled to iRGD bound to tumor vessels and spread into the extravascular tumor parenchyma, whereas conventional RGD peptides only delivered the cargo to the blood vessels. iRGD homes to tumors through a three-step process: the RGD motif mediates binding to $\alpha_{v}$ integrins on tumor endothelium, and a proteolytic cleavage then exposes a binding motif for neuropilin-1, which mediates penetration into tissue and cells. Conjugation to iRGD significantly improved the sensitivity of tumor-imaging agents and enhanced the activity of an antitumor drug.

\section{CONCLUSION}

Based on the information provided above, we propose an experimental study that will apply a strategy for peptide-mediated delivery of compounds deep into tumor parenchyma using tumor-homing peptides. Selected tyrosine kinase inhibitors such as imatinib will be coupled to tumor-specific homing peptides and will be used for the treatment of various GBM cell lines. Such targeted delivery of the antitumor agent can result in higher drug concentrations in tumors, increasing drug efficacy and reducing peripheral toxicity, thus overcoming the chemo-resistance of cancer cells, which is usually mediated by membrane transporters. This initiative is expected to result in new drug candidates by obtaining reliable data on the molecular pathogenesis of GBMs and molecular-targeted treatment options for GBMs. The development of drug candidates involving a delivery system based on previous knowledge and targeting intracellular pathways would facilitate the identification of more effective treatment options, thereby positively affecting the outcome, survival rate, and quality of life in patients with GBM.

\section{REFERENCES}

1. Sathornsumetee S, Reardon DA. Targeting multiple kinases in glioblastoma multiforme. Expert Opin Investig Drugs 2009; 18:277-92.

2. Argyriou AA, Antonacopoulou A, Iconomou G, Kalofonos HP. Treatment options for malignant gliomas, emphasizing towards new molecularly targeted therapies. Crit Rev Oncol Hematol 2009;69:199-210.

3. Maher EA, Furnari FB, Bachoo RM, Rowitch DH, Louis DN, Cavenee WK, DePinho RA. Malignant glioma: genetics and biology of a grave matter. Genes Dev 2001;15:1311-33.

4. Noda SE, El-Jawahri A, Patel D, Lautenschlaeger T, Siedow M, Chakravarti A. Molecular advances of brain tumors in radiation oncology. Semin Radiat Oncol 2009;19:171-8.

5. Argyriou AA, Kalofonos HP. Molecularly targeted therapies for malignant gliomas. Mol Med 2009;15:115-22.

6. Behin A, Hoang-Xuan K, Carpentier AF, Delattre JY. Primary brain tumours in adults. Lancet 2003;361:323-31.

7. Yeung YT, McDonald KL, Grewal T, Munoz L. Interleukins in glioblastoma pathophysiology: implications for therapy. $\mathrm{Br} J$ Pharmacol 2013;168:591-606.

8. Leon SP, Folkerth RD, Black PM. Microvessel density is a prognostic indicator for patients with astroglial brain tumors. Cancer 1996;77:362-72.

9. Bergers G, Benjamin LE. Tumorigenesis and the angiogenic switch. Nat Rev Cancer 2003;3:401-10.

10. Semenza GL. Vasculogenesis, angiogenesis, and arteriogenesis: mechanisms of blood vessel formation and remodeling. $J$ Cell Biochem 2007;102:840-7.

11. D'Abaco GM, Kaye AH. Integrins: molecular determinants of glioma invasion. J Clin Neurosci 2007;14:1041-8.

12. Cox BD, Natarajan M, Stettner MR, Gladson CL. New concepts regarding focal adhesion kinase promotion of cell migration and proliferation. J Cell Biochem 2006;99:35-52.

13. Hauck CR, Hsia DA, Schlaepfer DD. The focal adhesion kinase-a regulator of cell migration and invasion. IUBMB Life 2002;53:115-9.

14. Walsh EM, Kim R, Del Valle L, Weaver M, Sheffield J, Lazarovici P, Marcinkiewicz C. Importance of interaction between nerve growth 
factor and $\alpha 9 \beta 1$ integrin in glial tumor angiogenesis. Neuro Oncol 2012;14:890-901.

15. Dimitropoulos K, Giannopoulou E, Argyriou AA, Zolota V, Petsas T, Tsiata E, Kalofonos HP. The effects of anti-VEGFR and anti-EGFR agents on glioma cell migration through implication of growth factors with integrins. Anticancer Res 2010;30:4987-92.

16. Binder BR, Mihaly J, Prager GW. uPAR-uPA-PAI-1 interactions and signaling: a vascular biologist's view. Thromb Haemost 2007; $97: 336-42$

17. Desgrosellier JS, Cheresh DA. Integrins in cancer: biological implications and therapeutic opportunities. Nat Rev Cancer 2010;10:9-22 .

18. Xiong JP, Stehle T, Zhang R, Joachimiak A, Frech M, Goodman SL, Arnaout MA. Crystal structure of the extracellular segment of integrin alpha Vbeta3 in complex with an Arg-Gly-Asp ligand. Science 2002;296:151-5.

19. Nisato RE, Tille JC, Jonczyk A, Goodman SL, Pepper MS. alphav beta 3 and alphav beta 5 integrin antagonists inhibit angiogenesis in vitro. Angiogenesis 2003;6:105-19.
20. MacDonald TJ, Taga T, Shimada H, Tabrizi P, Zlokovic BV, Cheresh DA, Laug WE. Preferential susceptibility of brain tumors to the antiangiogenic effects of an alpha(v) integrin antagonist. Neurosurgery 2001;48:151-7.

21. Tabatabai G, Weller M, Nabors B, Picard M, Reardon D, Mikkelsen T, Ruegg C, Stupp R. Targeting integrins in malignant glioma. Target Oncol 2010;5:175-81.

22. Sugahara KN, Teesalu T, Karmali PP, Kotamraju VR, Agemy L, Girard OM, Hanahan D, Mattrey RF, Ruoslahti E. Tissue-penetrating delivery of compounds and nanoparticles into tumors. Cancer Cell 2009; 16:510-20.

Cite this article as: Giannopoulou E, Tzakos A, Argyriou AA. Integrins and focal adhesion kinase in the malignant behavior of gliomas. Neuroimmunol Neuroinflammation 2015;2(1):4-7.

Source of Support: Nil. Conflict of Interest: No.

Received: 01-09-2014; Accepted: 16-10-2014 